\title{
Enhanced Synaptic Integration of Adult-Born Neurons in the Olfactory Bulb of Lactating Mothers
}

\author{
Hagit Kopel, ${ }^{1,2}$ Eitan Schechtman, ${ }^{2}$ Maya Groysman, ${ }^{1,2}$ and Adi Mizrahi ${ }^{1,2}$ \\ ${ }^{1}$ Department of Neurobiology, Institute of Life Sciences, and ${ }^{2}$ The Edmond and Lily Safra Center for Brain Sciences, The Hebrew University of Jerusalem, \\ Edmond J. Safra Campus, Givat Ram, Jerusalem, 91904, Israel
}

One of the most dramatic events during the life of adult mammals is the transition into motherhood. This transition is accompanied by specific maternal behaviors, displayed by the mother, that ensure the survival and the well-being of her offspring. The execution of these behaviors is most likely accompanied by plastic changes in specific neuronal circuits, but these are still poorly defined. In this work, we studied the mammalian olfactory bulb $(\mathrm{OB})$, which has been shown to be an essential brain region for maternal behaviors in mice. In the $\mathrm{OB}$, we focused on adult-born neurons, which are continuously incorporated into the circuit during adulthood, thus providing a potential substrate for heightened plasticity after parturition. We analyzed the dynamics and morphological characteristics of adult-born granule cells (abGCs), innervating the $\mathrm{OB}$ of primiparous lactating mothers, shortly after parturition as well as in naive females. In vivo time-lapse imaging of abGCs revealed that dendritic spines were significantly more stable in lactating mothers compared with naive virgins. In contrast, spine stability of resident GCs remained unchanged after parturition. In addition, while spine size distribution of abGCs was approximately similar between mothers and naive virgins, the spine density of abGCs was lower in lactating mothers and the density of their presynaptic components was higher. These structural features are indicative of enhanced integration of adult-born neurons into the bulbar circuitry of lactating mothers. This enhanced integration may serve as a cellular mechanism, supporting changes in olfactory coding of new mothers during their first days following parturition.

\section{Introduction}

Across the animal kingdom, the transition into motherhood is accompanied by well characterized behaviors, which ensure reproductive success. In most mammals, this form of behavioral plasticity starts at parturition, or shortly thereafter, and is characterized by intensive protection and nursing of the offspring. In mice for example, maternal behavior is reflected in nest building, pup retrieval, pup licking and crouching (Noirot, 1969). While the transition into motherhood has been studied extensively at both the behavioral and the hormonal levels, the neuronal correlates of these changes remain largely unknown.

In many animal species, olfaction plays a central role in numerous basic functions; one of which is social recognition (Schaefer et al., 2002; Brennan and Kendrick, 2006; Baum and Kelliher, 2009; Keller et al., 2009). In the context of maternal behavior, the olfactory system may be particularly important for mothers to recognize their pups and to protect them from foes in the vicinity of the nest. Indeed, in mice, olfaction is essential

\footnotetext{
Received Dec. 21, 2011; revised March 20, 2012; accepted April 4, 2012.

Author contributions: H.K. and A.M. designed research; H.K. performed research; M.G. contributed unpublished reagents/analytic tools; H.K. and E.S. analyzed data; H.K. and A.M. wrote the paper.

This work was supported by a European Research Council grant to A.M. (Grant 203994). We thank Y. Livneh for his assistance throughout this project, S. Rumpel and Y. Loewenstein for advice on spine size analysis, I. Nelken for advice on statistical analysis, N. Melamed-Book for technical help with the confocal microscope, and the members of the Mizrahi Laboratory for discussions and comments on the manuscript.

The authors declare no competing financial interests.

Correspondence should be addressed to Adi Mizrahi at the above address. E-mail: mizrahia@cc.huji.ac.il.

DOI:10.1523/JNEUROSCI.6354-11.2012

Copyright $\odot 2012$ the authors $\quad 0270-6474 / 12 / 327519-09 \$ 15.00 / 0$
}

for mothers to behave maternally toward their offspring (Gandelman et al., 1971; Vandenbergh, 1973). Increasing evidence in numerous species suggest that neural plasticity, already at the level of the olfactory bulb $(\mathrm{OB})$, is important for maternal recognition and for the production of normal maternal behaviors (Dickinson and Keverne, 1988; Lévy et al., 1990; Kendrick et al., 1997; Sakamoto et al., 2011).

In the $\mathrm{OB}$, adult-born interneurons (abINs) are one neuronal population that can serve as a good candidate for the plasticity following parturition. In this respect, the abINs are an attractive population because they are continuously incorporated into the OB's circuitry in large numbers throughout adulthood (Altman, 1969; Lois and Alvarez-Buylla, 1994), forming a unique substrate for structural and functional plasticity (Lledo et al., 2006; Lazarini and Lledo, 2011). With regard to maternal care, it has been shown that in the absence of adult neurogenesis maternal behaviors are impaired (Sakamoto et al., 2011). Moreover adult neurogenesis, which is enhanced during pregnancy, is followed by a significant increase in the number of newly generated abINs in the OB (Shingo et al., 2003). The integration process of these neurons that were born during pregnancy is expected to occur during the time when parental care is essential and should be at its peak. The correlation between the increased surge of adult-born neurons integrating into the bulbar circuitry and parturition suggests that synaptic connections made by abINs are an integral part of $\mathrm{OB}$ plasticity during this time.

In this study, we characterized structural plasticity of abINs that were born during pregnancy and integrated into the $\mathrm{OB}$ circuitry during the first stages of motherhood. We focused on 
adult-born granule cells (abGCs), which are the major cell population that undergoes neurogenesis throughout adulthood in the OB (Lledo et al., 2006). Using in vivo time lapse two photon imaging as well as confocal imaging, we describe the structural synaptic properties of newly born neurons in the OB of lactating mothers. We show that abGCs' dendritic spines in mothers become stabilized and present enhanced integration compared with the same neuronal population in naive virgins. Our results reveal a new aspect of cellular plasticity unique to this early station of olfactory processing.

\section{Materials and Methods}

Animals. BALB/C mice ( $n=31$ mice, $10-14$ weeks old) were used in all experiments. The control group included naive virgin females that were never housed with males or pups after they had been weaned at postnatal day 21 (PD21). The experimental group included naive females that were placed in a cage with a proven breeder male. After $3 \mathrm{~d}$, the male was separated from the cage and after $7 \mathrm{~d}$, the females were injected with lentivirus (see below, Stereotaxic injection of lentivirus into the stem cell niche). This group consisted of lactating mothers that gave birth to at least three pups and displayed the following maternal behaviors: nursing, crouching and pup retrieval. Although litter size varied between the mice, we did not find a correlation between the mothers' litter size (as an indirect proxy for maternal behavior) (Seitz, 1958; Grota and Ader, 1969) and the structural changes in its neurons (data not shown). Therefore, all maternally behaving mothers were grouped into a single group. All experimental procedures used in this study were approved by the Hebrew University Animal Care and Use Committee.

Lentiviral vectors. To generate recombinant lentiviral vectors we subcloned a fusion protein of Synaptophysin and GFP (kind gift from D. Gitler, Ben Gurion University of the Negev, Israel) into a lentivirus transfer plasmid under the control of the elongation factor (EF)-1 $\alpha$ promoter. In addition, to express GFP we used transfer plasmids with GFP under the control of the CMV promoter or synapsin promoter. Virus was produced by transfection of human embryonic kidney cells (HEK293) with third-generation lentivirus plasmids using polyethylenimine. The medium was collected after $36 \mathrm{~h}$ and again after additional $24 \mathrm{~h}$. Virus was concentrated using ultracentrifugation and resuspended in PBS.

Stereotaxic injection of lentivirus into the stem cell niche. Lentivirus injections into the subventricular zone (SVZ) and into the rostral migratory stream (RMS) were performed as described earlier (Mizrahi, 2007). Briefly, mice were anesthetized using ketamine $(100 \mathrm{mg} / \mathrm{kg})$ and medetomidine $(0.83 \mathrm{mg} / \mathrm{kg})$. Carpofen $(0.004 \mathrm{mg} / \mathrm{g})$ was injected subcutaneously before surgery. One of the following lentiviruses was used to transduce newborn neurons: lentivirus encoding for GFP driven by CMV promoter and lentivirus encoding for Synaptophysin-GFP (Syn-GFP), driven by the EF-1- $\alpha$ promoter. Virus injections $(0.2-0.5 \mu \mathrm{l})$ were done stereotaxically into one hemisphere, using pressure (coordinates relative to bregma; SVZ: anterior, $1 \mathrm{~mm}$; lateral, $1 \mathrm{~mm}$; depth, $2.2 \mathrm{~mm}$; RMS: anterior, $3.3 \mathrm{~mm}$; lateral, $0.8 \mathrm{~mm}$; depth, $2.9 \mathrm{~mm}$ ). After surgery, mice fully recovered and were returned to the animal facility under normal housing conditions. Both naive and pregnant females returned to their original cages at groups of $2-3$ females. Approximately $8 \mathrm{~d}$ later $(\sim 2 \mathrm{~d}$ before parturition), mice from both groups were placed in separated cages until imaging or perfusion.

Chronic window implantation over both OBs. For the long term imaging experiment of resident GCs we used the cranial window preparation, which was recently described in detail (Adam and Mizrahi, 2011). Briefly, the skull overlying both OBs was carefully removed, leaving the dura intact. Lentivirus-GFP driven by synapsin promoter was injected into both bulbs to transduce resident neurons in the OB. Then, a $3 \mathrm{~mm}$ diameter circular glass coverslip (No. 1) was positioned over the opening and sealed in place using histoacryl (TissueSeal) and dental cement. A $0.1 \mathrm{~g}$ metal bar was glued to the skull for repositioning the animal's head under the microscope in consecutive imaging sessions (Mizrahi and Katz, 2003). After surgery, mice fully recovered and returned to the animal facility under normal housing conditions. Approximately 8 weeks later, mice were anesthetized and underwent the first imaging session with no further surgical interference (see below, In vivo two-photon imaging).

In vivo two-photon imaging. Time lapse imaging of abGCs in lactating mothers was performed at $3 \mathrm{~d}$ and at $4 \mathrm{~d}$ following parturition. This time corresponded to $14-17 \mathrm{~d}$ post-injection (d.p.i.) at the first imaging session. The naive females group was also imaged at $14-17$ d.p.i. (at the first imaging session). Imaging was performed in anesthetized freely breathing mice through a small craniotomy as described earlier (Mizrahi, 2007; Livneh et al., 2009). Time lapse imaging of resident GCs was performed four times (two times before, and two times after pregnancy, see Fig. 5A) through the implanted cranial window. In both experiments, the mouse was placed under the microscope in a custom-made stereotaxic device via a metal bar glued to the skull in a fixed orientation relative to the objective lens. Mice were imaged using a two-photon microscope (Prairie Technologies) equipped with a $40 \times(0.8 \mathrm{NA})$ IR-Achroplan waterimmersion objective (Olympus). A femtosecond laser (Mai-Tai Spectra Physics) was used to excite GFP at 900-920 nm. Images $(512 \times 512$ pixels) were acquired at $0.23 \mu \mathrm{m}$ per pixel resolution in the $X Y$ dimension and $0.9 \mu \mathrm{m}$ steps in the $Z$ dimension. Each dendritic tree was identified in the additional imaging sessions by its location in 3D relative to the blood vessel map (Mizrahi, 2007; Livneh et al., 2009).

Histology and tissue processing. Fifteen to 18 d.p.i. (corresponding to day 4 following parturition in the lactating mothers group), mice were perfused transcardially with $0.9 \%$ saline followed by $4 \%$ paraformaldehyde and the brains were cryoprotected in 30\% sucrose. OBs were sectioned coronally $(40 \mu \mathrm{m})$ on a sliding microtome. Syp-GFP was then amplified as described by others (Kelsch et al., 2008). Briefly, slices were incubated overnight with a primary antibody (rabbit anti-GFP, 1:1000, Millipore), and then with a secondary antibody (goat anti-rabbit-Cy3, 1:500, Jackson ImmunoResearch) for $3 \mathrm{~h}$. Both antibodies were diluted in blocking solution (5\% heat inactivated normal goat serum mixed with $0.5 \%$ Triton-X in PBS), and incubations were done in room temperature.

Confocal microscopy. Slices were imaged at $0.165-0.2 \mu \mathrm{m} / \mathrm{pixel}$ in the $X Y$ dimension and at $0.5 \mu \mathrm{m}$ steps in the $Z$ dimension. Imaging was performed with an Olympus FV-1000 confocal microscope, via a $40 \times$ (1.3 NA) oil objective or with a Leica SP5 confocal microscope via a $40 \times$ (1.25 NA) oil objective. We imaged and analyzed only distal GC dendrites in the external plexiform layer (EPL), as different parts of the GC dendritic tree were shown to have different properties (Kelsch et al., 2008, 2009). GFP expression levels were variable between neurons. Thus, we limited our analysis to the brightest neurons in the sample. This bias to high contrast signal may be one reason for the higher average spine densities than what we and others have reported earlier (Whitman and Greer, 2007; Dahlen et al., 2011; Livneh and Mizrahi, 2011; but see also Breton-Provencher et al., 2009; Lin et al., 2010). Another possible reason for the higher average spine densities is the way we scored spines with multiple heads (see below, Data analysis).

Data analysis. All analyses in this study were done blind to the experimental condition. Quantitative analyses of spine dynamics were performed manually from the filtered image stacks (Gaussian blur filter) using ImageJ (http://rsb.info.nih.gov/ij/). The imaged regions in the two sessions were aligned to each other using the "Sync windows" plugin (http://rsb.info.nih.gov/ij/plugins/sync-windows.html). Each spine was then scored as stable, lost, or gained using the "cell counter" plugin (http://rsb.info.nih.gov/ij/plugins/cell-counter.html). For each cell, spine dynamics were calculated as follows: number of stable spines $=$ $\mathrm{N}_{\text {stable }} /\left(\mathrm{N}_{\text {stable }}+\mathrm{N}_{\text {lost }}\right)$, number of lost spines $=\mathrm{N}_{\text {lost }} /\left(\mathrm{N}_{\text {stable }}+\mathrm{N}_{\text {lost }}\right)$, number of gained spines $=\mathrm{N}_{\text {gained }} /\left(\mathrm{N}_{\text {stable }}+\mathrm{N}_{\text {gained }}\right)$. $\mathrm{N}_{\text {stable }}, \mathrm{N}_{\text {lost }}$, and $\mathrm{N}_{\text {gained }}$ are the number of stable, lost, and gained spines, respectively.

Dendrites and spines were analyzed only if they had strong fluorescent signal that clearly contrasted from the background. To estimate the size of a spine, we used the integrated fluorescence of a spine head, which has been shown to be a good proxy of its size (Holtmaat et al., 2005). Analysis was performed by using custom software written in Matlab (MathWorks). First, each frame of the stack was filtered with a $3 \times 3$ pixel median filter. Then, for each spine its head, adjacent background, and shaft regions were marked manually (see Fig. 2C). Finally, spine head size was calculated in two dimensions at the image frame in which the integrated fluorescent of a single spine head was highest. Intensity values of 
all pixels comprising the spine head were summed after subtracting the mean value of the background adjacent to the spine. To normalize this value and to avoid biases that can be caused by inhomogeneities in excitation level and GFP expression, the integrated value of the fluorescence of a single spine head was divided by averaging the intensity levels of the pixels on the adjacent dendritic shaft, whose intensity level was on the upper $10 \%$ of that dendrite. The result of this analysis was the spine head size having arbitrary units (AU). Higher values of this measure reflected bigger spine head sizes. In the current dataset, spines ranged from 0.15 to $43 \mathrm{AU}$ (see Fig. $2 C$ for examples).

GFP-based spine density and Syp cluster density were calculated from $3 \mathrm{D}$ reconstructions of arbitrarily chosen dendritic trees within the EPL, belonging to the same neuron. Three-dimensional reconstructions were performed manually using Neurolucida (MicrobrightField) strictly from the raw data images as we described earlier for PSD95-GFP puncta (Livneh et al., 2009). In several cases we observed multiple spines emerging from the same spine neck or parent dendrite. In those cases, all spine heads were counted as spines, regardless of the neck origin.

Statistical analysis. Statistical analyses were performed using Matlab (MathWorks). For spine dynamics, the nonparametric Mann-Whitney $U$ test was used. For spine size, spine density and Syp-GFP cluster density analyses the $n$-way nested ANOVA test was used. In all statistical tests, unless otherwise mentioned, significance was accepted at $p=0.05$, and data are presented as mean \pm SEM.

\section{Results}

To study synapses of abGCs of mothers, we transduced new neurons with lentivirus encoding for green fluorescent protein (GFP) during pregnancy and imaged their development shortly following parturition. We compared a few structural properties between abGCs in primiparous lactating mothers (the experimental group) and age-matched naive virgins (the control group). First, we studied short-term spine dynamics of abGCs, using in vivo time lapse imaging as a measure of their general plasticity (Bhatt et al., 2009; Holtmaat and Svoboda, 2009; Hübener and Bonhoeffer, 2010; Roberts et al., 2010). Then, we studied spine size distribution, using confocal imaging, as a measure of their synaptic strength. Finally, we studied abGCs' spine density as well as the density of their presynaptic components as a measure of their developmental maturation and functional integration into the OB circuit.

\section{Increased spine stability in lactating mothers}

To express GFP in adult-born neurons we used lentivirus injections into the stem cell niche (both RMS and SVZ). Newborn neurons were transduced during pregnancy at gestation day 8 (GD8 \pm 1$)$, and then imaged twice, at day 3 and at day 4 following parturition (PD3, PD4; Fig. 1A). Using this experimental configuration, imaging days corresponded to neuronal age of 14-17 d.p.i. at the first imaging session in both groups. Fourteen to seventeen d.p.i. is a time window when young abGCs already have branched dendritic trees in the EPL, which are studded with many spines. As characterized by physiological and morphological criteria, this morphology corresponds to the final stage of development, known as "stage 5" (Petreanu and Alvarez-Buylla, 2002; Carleton et al., 2003). We first evaluated the structural plasticity of stage 5 abGCs in both lactating mothers and naive virgins by quantifying the $24 \mathrm{~h}$ spine turnover rate using timelapse two photon imaging, in vivo.

As a measure of plasticity we quantified each spine as stable, lost, or gained (Fig. $1 B, D)$. We calculated each neuron's turnover rate [TOR; TOR $\left.=\left(\mathrm{N}_{\text {lost }}+\mathrm{N}_{\text {gained }}\right) /\left(\mathrm{N}_{\text {total 1st session }}+\mathrm{N}_{\text {total } 2 \text { nd session }}\right)\right]$, which measures the overall structural dynamics of the cells (Holtmaat et al., 2009). Higher values of TOR reflect higher spine dynamics. In both groups, the fraction of lost spines was not significantly dif-
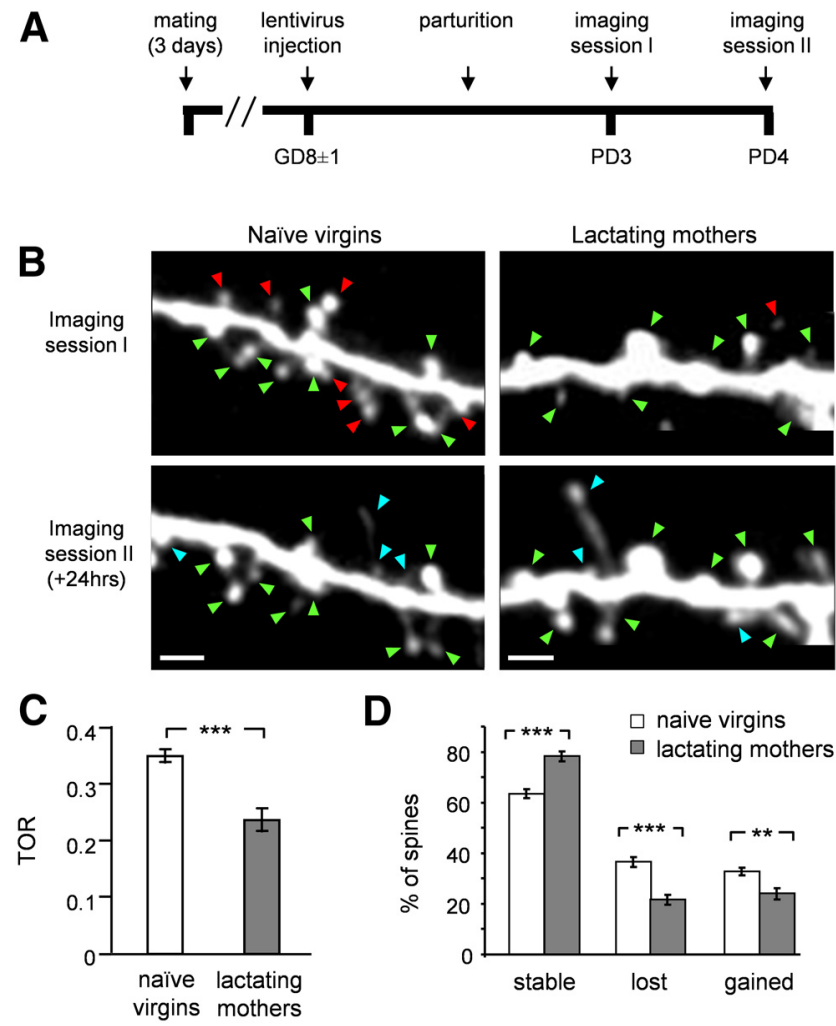

Figure 1. Spines of abGCs stabilize following parturition. $\boldsymbol{A}$, The time line of the experimental protocol in lactating mothers. Naive virgins were injected with lentivirus and imaged at the same day post-injection as in the experimental group. $\boldsymbol{B}$, Projection images of abGCs' dendritic spines imaged in vivo at $24 \mathrm{~h}$ interval in naive virgins (left) and lactating mothers (right). Stable, lost, and gained spines are marked by green, red, and blue arrowheads, respectively. Scale bars: $5 \mu \mathrm{m}$. C, D, Quantitative analysis of abGC's spine dynamics (naive virgins: $n=873$ spines, from 11 cells in 6 mice; lactating mothers: $n=591$ spines, from 11 cells in 6 mice). C, Mean turnover rate (TOR) of abGCs spines from naive virgins and lactating mothers. $\boldsymbol{D}$, Mean percentage of stable, lost, and gained spines in the two groups. All values are mean \pm SEM; ${ }^{* *} p<0.01$, ${ }^{* * *} p<0.001$, Mann-Whitney $U$ test.

ferent from the fraction of gained spines, suggesting that cells in both groups have ceased accumulating synapses, at least within a $24 \mathrm{~h}$ time frame (Fig. $1 D ; p=0.149$ in naive virgins group, $p=$ 0.375 in lactating mothers group; Mann-Whitney $U$ test). In control mice (i.e., naive virgins), spines of young abGCs were highly dynamic (Fig. $1 B$, left). TOR levels reached $0.34 \pm 0.01$ (Fig. $1 C$, open bar). Accordingly, only $\sim 60 \%$ of the spines remained stable over a $24 \mathrm{~h}$ time window (Fig. $1 \mathrm{D}$ ). In contrast, spines of abGCs in lactating mothers were significantly more stable (Fig. $1 \mathrm{~B}$, right). The average TOR levels significantly decreased to $0.23 \pm 0.02$ on average and almost $80 \%$ of spines were stable within a $24 \mathrm{~h}$ time frame (Fig. 1C,D, filled bars). Overall, lactating mothers had significantly reduced spine dynamics compared with naive virgins in all measures of spine dynamics (TOR: $p=0.00043$, stable spines: $p=0.00056$, lost spines: $p=0.00056$, and gained spines: $p=0.0058$; Mann-Whitney $U$ test).

Increased spine stability is a characteristic of maturing neurons (Grutzendler et al., 2002; Zuo et al., 2005; Alvarez and Sabatini, 2007; Mizrahi, 2007). Thus, we next asked whether the changes in spine dynamics are also accompanied by other morphological differences, pointing to differential development of adult-born neurons in mothers. To test this possibility, we analyzed in more detail two additional morphological parameters of abGC synapses: spine size and spine density, as described next. 
Spine size and spine density of abGCs in lactating mothers and naive virgins

To study spine morphology of abGCs we used a similar experimental procedure to the one described above, but now fixed the brains at PD4 (and on corresponding days in the control group). We then imaged the morphology of abGCs using high-resolution confocal microscopy (Fig. $2 A, B$ ). Consistent with stage 5 neurons, abGCs had well developed dendritic arbors studded with many spines (Fig. $2 B$ ). Because different parts of the GCs' dendritic tree may have different properties (Kelsch et al., 2008, 2009), we focused our analysis of abGCs' spine morphology to their distal apical dendrites within the EPL, where they form reciprocal synapses with mitral cells (MCs) — the bulb's main projection neurons.

As expected, spines of abGCs were structurally heterogeneous (Fig. 2C, top). Some spines had long necks, others were "stubby" (i.e., no visible neck), and yet others had neck lengths that may fall anywhere in between. Some spiny protrusions ended with only one spine head while others had multiple heads (Fig. 2C, top). Here, we limited our analysis regarding the shape of the spines to a measure of spine head size; a measure used for synaptic strength and synaptic plasticity in other brain systems (Matsuzaki et al., 2004; Kopec et al., 2006; Hofer et al., 2009; Roberts et al., 2010). To this end, we calculated spine head sizes semiautomatically by manually marking each spine head, its adjacent background and its adjacent dendritic shaft. Then, the spine head size was calculated automatically from the 3D stack (Fig. 2C, bottom; see also Materials and Methods, Data analysis, for details of this analysis).

Spine head size distributions of abGCs varied greatly in both naive virgins and lactating mothers (Fig. 2D). We found no significant differences between the distributions of the two experimental groups (Fig. $2 D ; p=0.147, n$-way nested ANOVA test). Nevertheless, we could detect a small difference in the upper tail of the distributions. Specifically, comparing the distributions of the 75th percentiles of the cells within each group, revealed that these values were slightly lower in lactating mothers $(p=0.04, n$-ways nested ANOVA test). This result may point to a small population of large spines, which is evident only in the control group. Notably, however, neither the 25 th nor the 50th percentiles were significantly different between lactating mothers and naive virgins (25th percentile: $p=0.36$; 50th percentile: $p=0.14, n$-ways nested ANOVA test). Thus, we concluded that there is no robust difference in spine head sizes between lactating mothers and naive virgins.

The next morphological parameter we tested was abGCs' spine density, a measure that can indicate the maturation level of a neuron. This analysis was done using a similar dataset that we acquired for the spine size data above and was performed manually on the 3D stack from each neuron's dendritic tree. Although spine density values varied widely between cells within each group (Fig. $3 A$ ), the mean spine density of abGCs was significantly lower in lactating mothers compared with naive virgins (Fig. $3 B ; p=0.034, n$-way nested ANOVA test). In the developing brain it is well established that there is a rapid increase in spine density during early development, which is followed by a prolonged synapse elimination period (Rakic et al., 1986; Grutzendler et al., 2002; Holtmaat et al., 2005; Zuo et al., 2005; Alvarez and Sabatini, 2007). This trend of overproduction followed by a decrease in the density has also been shown during abGCs' development in the adult OB (Whitman and Greer, 2007; Livneh and Mizrahi, 2011). Our results show that abGCs in lactating mothers 
A

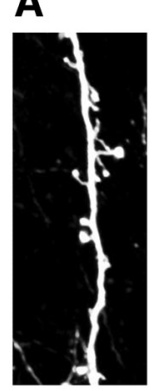

0.34

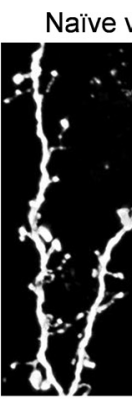

0.39

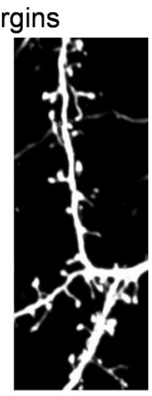

0.6

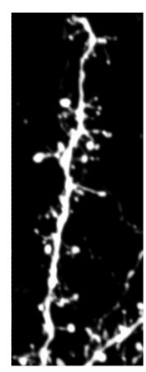

0.75

spines/ $\mu \mathrm{m}$

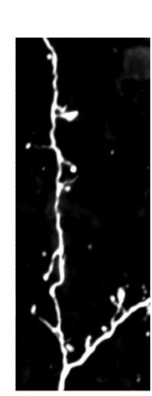

0.17

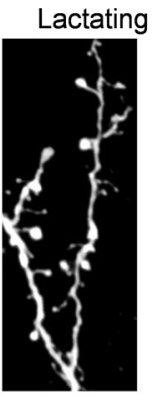

0.3

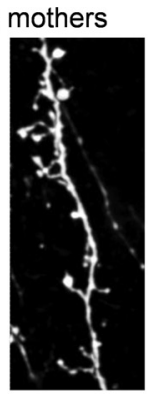

0.4

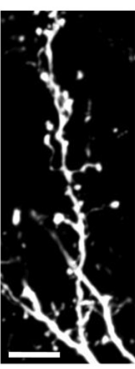

0.52 spines/ $\mu \mathrm{m}$

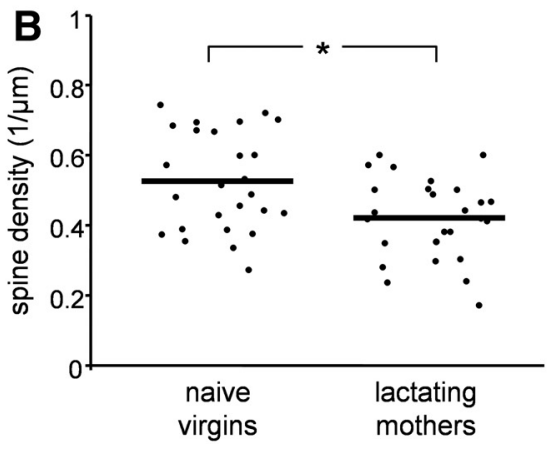

Figure 3. Spine density of abGCs is lower in lactating mothers following parturition. A, Projection images of abGC spiny dendrites from naive virgins (left) and lactating mothers (right). All examples were taken from the EPL. The number below each panel is the spine density calculated from the $3 \mathrm{D}$ stack of this specific segment (units are in spines/ $\mu \mathrm{m}$ ). Examples are in ascending order for clarity. Scale bar: $10 \mu \mathrm{m} . \boldsymbol{B}$, Quantitative analysis of spine density in naive virgins and lactating mothers. Each dot represents the spine density of an individual cell. The mean value of each group is indicated by the horizontal line. Naive virgins: $n=26$ cells from 6 mice; lactating mothers: $n=26$ cells from 6 mice. A total of 6676 clusters were analyzed. ${ }^{*} p=0.034, n$-way nested ANOVA test.

are at a different developmental stage compared with the same neuronal population in naive females. However, lower spine densities can be indicative of either delayed maturation or enhanced maturation, depending on the exact developmental state of the cell. Therefore, to further investigate abGCs maturation, we next quantified the level of their synaptic integration in both lactating mothers and naive females.

Higher density of presynapses in abGCs of lactating mothers During development, the appearance of abGCs dendritic spines can occur before they express synaptic markers (Whitman and Greer, 2007). Thus, in young developing neurons spine density alone cannot provide a comprehensive measurement for the level of synaptic integration. Since the density of synaptic markers (both the postsynaptic and the presynaptic components) increases during development (Whitman and Greer, 2007), they can be used as one measure of the level of abGCs' synaptic integration within the EPL. To complement our spine density analysis in the context of abGCs' synaptic output, we next tested the density of their presynaptic component in the same neuronal age.

To study the development of presynaptic components in abGCs, we expressed the synaptic vesicle protein, synaptophysin, fused to GFP (Syp-GFP). Injections of lentivirus encoding SypGFP into the stem cell niche resulted in abGCs having fluorescent clusters of synaptophysin (SypC) along their dendritic tree; a correlate of their presynaptic components on dendrodendritic synapses (Kelsch et al., 2008). To reveal both the dendritic morphology and synaptic puncta of abGCs, we amplified their GFP levels by immunohistochemistry (Fig. 4A; Kelsch et al., 2008). The criterion for identifying a SypC was colocalization of a yellow punctum within a spine head (i.e., green puncta that were not colocalized with a spine head were not analyzed). In contrast to the spine density results, the mean density of SypC in lactating mothers was $\sim 30 \%$ higher than that of naive virgins (Fig. $4 B$; SypC density per $\mu \mathrm{m}: 0.315$ in naive females and 0.406 in lactating mothers; $p=0.0044 ; n$-way nested ANOVA test). These results of increased SypC density in lactating mothers, in combination with decreased spine dynamics and decreased spine densities, suggest that abGCs of lactating mothers undergo enhanced integration into the bulbar network.

\section{Spine dynamics of resident GCs do not change}

\section{following parturition}

The results described above might not be specific to abINs. Indeed, this structural plasticity could represent a general form of plasticity, which occurs in the general granule cell (GC) population within the $\mathrm{OB}$ of lactating mothers. To test this possibility, we studied the $24 \mathrm{~h}$ spine dynamics of resident GCs both before pregnancy and after parturition. For this purpose, we injected lenti-synapsin-GFP into the OB of naive females and implanted a chronic cranial window suitable for long term imaging (Adam and Mizrahi, 2011). A few weeks later, labeled GCs were imaged at a $24 \mathrm{~h}$ interval. Then, these naive females were mated and three and four days following parturition the same GCs were imaged again. With this experimental design, the same neurons were used as their own controls (i.e., before pregnancy) to compare with their behavior after pregnancy (Fig. 5A). As expected, spines of resident GCs were more stable than young abGCs, reaching levels of $\sim 88 \%$ stability in their $24 \mathrm{~h}$ dynamics (compare to Fig. $1 \mathrm{D}$, naive virgins). Interestingly, these values did not change following parturition (Fig. 5B,C). Thus, resident GCs following parturition were as stable as they were before pregnancy. These results suggest that the structural plasticity observed in abGCs of 

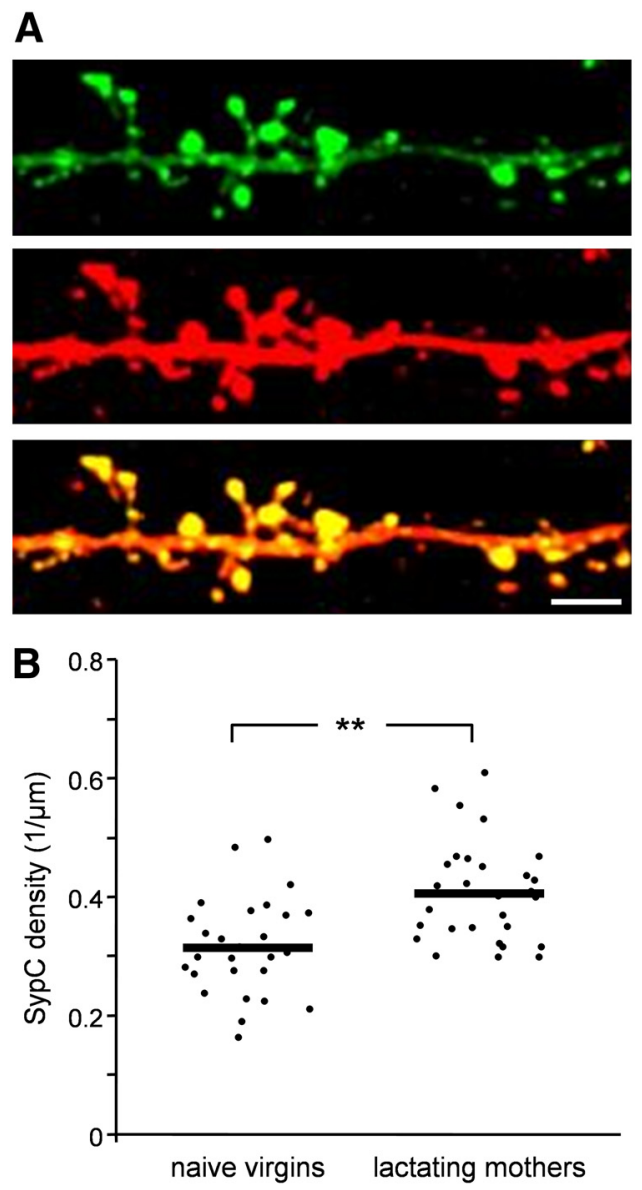

Figure 4. SypC density of abGCs is higher in lactating mothers following parturition. $\boldsymbol{A}$, Representative projection image of an abGC dendritic branch from naive virgin expressing SypGFP (top), amplified by an anti-GFP antibody (middle) and a merged image (bottom). Scale bar: $5 \mu \mathrm{m}$. B, Quantitative comparison of SypC density between naive virgins and lactating mothers. Each dot represents the SypC density of a single cell. The mean value of each experimental group is indicated by the horizontal black line. Naive virgins: $n=27$ cells from 6 mice; lactating mothers: $n=26$ cells from 5 mice. A total of 6950 spines were analyzed. ${ }^{* *} p<0.001, n$-way nested ANOVA test.

lactating mothers cannot be interpreted as a generalized stabilization shared across the GC population.

\section{Discussion}

In this study we found that abGCs in lactating mothers show unique structural characteristics compared with age-matched neurons in naive females. Specifically, abGCs' dendritic spines were more stable in lactating mothers shortly after parturition. Concomitantly, abGCs' spine density was lower and SypC density was higher in lactating mothers. No significant change in the spine size distribution was evident between the groups. These results suggest that abGCs that were born during pregnancy undergo enhanced integration into the bulbar circuitry following parturition. The timing of this enhanced integration suggests a scenario whereby changes in the GC-MC synapse of abINs is one of the cellular mechanisms underlying the behavioral plasticity in new mothers.

Possible contribution of the enhanced integration of abGCs to maternal behavior and to olfactory coding Our data provide evidence for unique changes in adult-born neurons during the transition into motherhood. One implication of

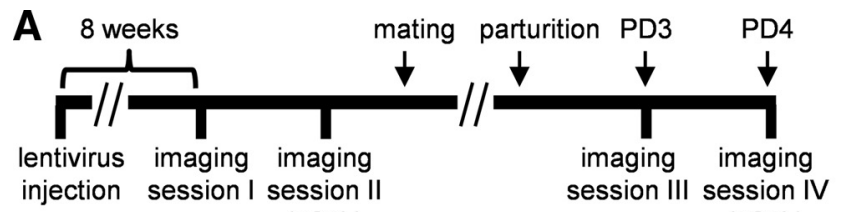

$(+24 h)$

$(+24 h)$

\section{B}

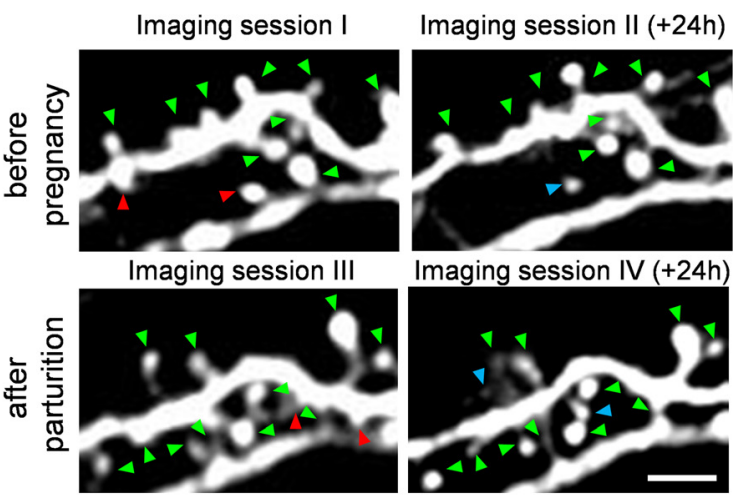

C

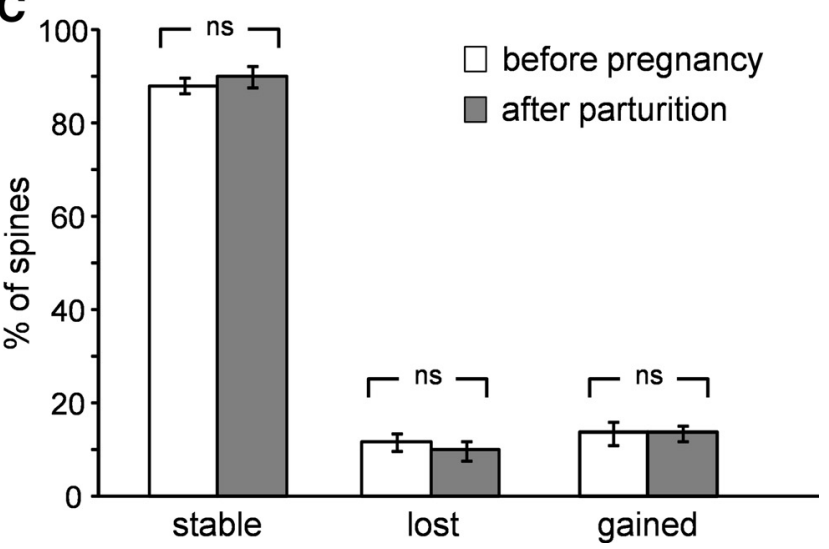

Figure 5. Resident $\mathrm{GCs}$ do not show changes in spine dynamics after parturition. $\boldsymbol{A}$, The time line of the experimental protocol. $\boldsymbol{B}$, Projection images of resident GCs imaged in vivo at $24 \mathrm{~h}$ interval before pregnancy (top) and after parturition (bottom). Stable, lost, and gained spines are marked by green, red, and blue arrowheads, respectively. Scale bars: $5 \mu \mathrm{m}$. C, Quantitative analysis of resident $\mathrm{GC} \mathrm{s}^{\prime}$ dynamics showing no change in stable lost and gained spines. $N=441$ spines, from 6 cells in 2 mice. All values are mean \pm SEM, Mann-Whitney $U$ test.

our results would be that adult neurogenesis plays a role during this period of time. Indeed, the role of adult neurogenesis in motherhood has been demonstrated by a recent study (Sakamoto et al., 2011). Sakamoto and colleagues used genetic tools to ablate newborn neurons in adult mice. This procedure of massive reduction of neurogenesis resulted in severely impaired nursing behaviors by the new mothers. Specifically, mothers failed to feed their own pups, did not retrieve them, and most pups died within $24 \mathrm{~h}$ of delivery. However, in another recent study, when neurogenesis was reduced by focal irradiation (and thus to a lesser extent compared with the genetic ablation), mothers recognized their own pups successfully and maternal behaviors were largely unaffected (Feierstein et al., 2010). Notably, the different methods used to decrease neurogenesis in these two studies induced very different effects not only on the newborn population, but perhaps also on the intact granule cell population. Therefore, the results of these studies should be compared with caution. In addition, since it has been shown previously that there is a compensatory behavior by granule cells in the $\mathrm{OB}$ when neurogenesis levels are decreased (Saghatelyan et al., 2005), further studies are 
still desired to evaluate the exact contribution of adult newborn neurons' integration and survival to maternal behavior.

How do GC-MC synapses impact olfactory coding and behavior? GCs form reciprocal synapses with MCs. MCs activity causes excitation of GCs, which in turn inhibit MCs, by releasing GABA (Mori and Takagi, 1978; Jahr and Nicoll, 1980; Shepherd et al., 2004). This GABAergic inhibition is thought to underlie recurrent and lateral inhibition within the OB (Isaacson and Strowbridge, 1998; Mori et al., 1999; Margrie et al., 2001; Urban and Arevian, 2009). Several partially overlapping functions have been attributed to GC inhibition in the OB. For example, GCs have been implicated in generating MC oscillatory synchronization (Laurent et al., 2001; Nusser et al., 2001), sharpening odor representation (Yokoi et al., 1995; Urban, 2002) and even supporting olfactory learning and memory (Kendrick et al., 1992; Brennan et al., 1995, 1998). Behaviorally, enhancement of GC inhibition in mice resulted in improved olfactory discrimination (Abraham et al., 2010). Thus, GC inhibition seems central for odor coding in the $\mathrm{OB}$ and contributes to the animal's ability to discriminate between different stimuli.

Could GC synapses also be involved in olfactory plasticity during motherhood? Our structural data (Fig. 4) predicts that abGCs' inhibition onto MCs will be increased following parturition. In line with this hypothesis, seminal work in sheep showed that basal release of GABA in the OB is increased following parturition (Kendrick et al., 1992). Moreover, the offspring's selective recognition by the mothers was correlated to the amount of GABA release. Specifically, salient stimuli such as the mother's own offspring induced higher levels of GABA release compared with those induced by odors of alien offspring (Kendrick et al., 1992). Although these experiments were performed in sheep, our results from mice suggest that the newborn neurons are prominent candidates for this type of enhanced inhibition in the $\mathrm{OB}$ following parturition.

The morphological plasticity that we uncover here is part of the normal repertoire of adult-born neurons following parturition with or without regard to survival rates. Although the relationship between the number of surviving adult-born neurons and the levels of plasticity that each neuron undergoes is still an open question, our data introduce an additional possible mechanism of plasticity operating in the $\mathrm{OB}$ of new mothers. This mechanism (i.e., enhanced synaptic integration) should be considered in models of maternal plasticity regardless of the total number of new neurons (Shingo et al., 2003; Feierstein et al., 2010).

\section{Regulation of integration of adult-born neurons in normal and maternal states}

What factors might regulate the enhanced integration of abGCs, which we observed? One prominent factor is sensory experience. Indeed, the central role of sensory experience in regulating the integration and maturation of adult-born neurons is supported by ample evidence. While sensory enrichment increases adult born neurons' survival rate (Rochefort et al., 2002), sensory deprivation decreases it (Petreanu and Alvarez-Buylla, 2002; Yamaguchi and Mori, 2005). In the context of synaptogenesis, our previous imaging studies have shown that enhanced sensory stimulation increases dendritic development and synaptic integration of adult-born neurons (Livneh et al., 2009). In line with these results, others have shown that sensory deprivation can reduce synaptic integration of adult-born neurons (Kelsch et al., 2009). During the transition into motherhood, the sensory environment of mothers certainly undergoes significant changes.
New mothers become continuously exposed to a variety of new odors, particularly those arising from the pups. Interestingly, naive virgins that cared for pups started to display maternal behaviors and also showed changes in sensory coding (Noirot, 1972; Cohen et al., 2011). Thus, one possibility is that the exposure to the new olfactory environment regulates the enhanced integration of adult-born neurons in mothers following parturition.

In addition to the external changes in the vicinity of the new mothers, they also undergo significant internal changes following pregnancy and parturition (Carter et al., 2001; Mann and Bridges, 2001; Brunton and Russell, 2008). Specifically, the hormonal balance changes dramatically in females during pregnancy, at parturition, and shortly afterward. Interestingly, many of the relevant hormones that undergo these changes are also known to regulate adult neurogenesis in general, and in the $\mathrm{OB}$ in particular (Lévy et al., 2011). For example, high levels of estradiol downregulate cell proliferation in the SVZ and consequently the number of adult-born neurons in the OB (Brock et al., 2010). Prolactin concentrations have been shown to be correlated with levels of proliferation in the SVZ and with the total number of adult-born neurons surviving in the OB (Shingo et al., 2003; Larsen and Grattan, 2010). Whether sensory environment, hormonal control or both regulate the structural changes we report here remains an open question.

\section{Plasticity beyond abGCs}

The transition into motherhood is a complex process, which is supported by neuronal changes beyond the $\mathrm{OB}$ and beyond olfaction (Liu et al., 2006; Galindo-Leon et al., 2009; Leuner et al., 2010; Leuner and Gould, 2010; Cohen et al., 2011). Even structural remodeling in the context of motherhood has been described in other brain areas. For example, CA1 pyramidal neurons of the hippocampus and pyramidal neurons of the medial prefrontal cortex show increased spine densities at late pregnancy and/or following parturition (Kinsley et al., 2006; Leuner and Gould, 2010). In addition, dendritic morphology (i.e., dendritic length and number of branch points) has been shown to be modulated by motherhood in both CA1 and CA3 neurons (Pawluski and Galea, 2006). Particularly relevant are changes in the hippocampus, which is the other main neurogenic region of the mammalian brain (Altman and Das, 1965; Guéneau et al., 1982; Gross, 2000; Ming and Song, 2005). Although no cell proliferation was reported during pregnancy in the hippocampus (Shingo et al., 2003; Furuta and Bridges, 2005; Pawluski et al., 2010), the possibility of heightened neuronal plasticity of adult generated neurons remains an open matter to explore.

Structural plasticity in the context of motherhood in other brain regions prompts the question whether resident GCs or other neurons in the $\mathrm{OB}$ also show structural changes in mothers. Resident GCs showed no significant differences in their $24 \mathrm{~h}$ spine dynamics tested before pregnancy and following parturition suggesting that our results are not a general phenomenon of all neurons in the $\mathrm{OB}$ (Fig. 5). However, it is well possible that resident GCs show other forms of plasticity (Gao and Strowbridge, 2009). In fact, the adult-born neurons themselves have been shown to exhibit different types of plasticity as they mature, as was recently shown using sensory enrichment (Livneh and Mizrahi, 2012).

In conclusion, we show that the transition into motherhood is accompanied by changes in the morphology of newborn neurons in the OB. In particular, our data suggest that abGCs undergo enhanced integration into the circuitry of new mothers. This enhanced integration of adult-born neurons can contribute to ol- 
factory coding of the mothers and may have direct impact on mother-pup interaction.

\section{References}

Abraham NM, Egger V, Shimshek DR, Renden R, Fukunaga I, Sprengel R, Seeburg PH, Klugmann M, Margrie TW, Schaefer AT, Kuner T (2010) Synaptic inhibition in the olfactory bulb accelerates odor discrimination in mice. Neuron 65:399-411.

Adam Y, Mizrahi A (2011) Long-term imaging reveals dynamic changes in the neuronal composition of the glomerular layer. J Neurosci 31:79677973.

Altman J (1969) Autoradiographic and histological studies of postnatal neurogenesis. IV. Cell proliferation and migration in the anterior forebrain, with special reference to persisting neurogenesis in the olfactory bulb. J Comp Neurol 137:433-457.

Altman J, Das GD (1965) Autoradiographic and histological evidence of postnatal hippocampal neurogenesis in rats. J Comp Neurol 124:319335.

Alvarez VA, Sabatini BL (2007) Anatomical and physiological plasticity of dendritic spines. Annu Rev Neurosci 30:79-97.

Baum MJ, Kelliher KR (2009) Complementary roles of the main and accessory olfactory systems in mammalian mate recognition. Annu Rev Physiol 71:141-160.

Bhatt DH, Zhang S, Gan WB (2009) Dendritic spine dynamics. Annu Rev Physiol 71:261-282.

Brennan PA, Kendrick KM (2006) Mammalian social odours: attraction and individual recognition. Philos Trans $\mathrm{R}$ Soc Lond B Biol Sci 361:2061-2078.

Brennan PA, Kendrick KM, Keverne EB (1995) Neurotransmitter release in the accessory olfactory bulb during and after the formation of an olfactory memory in mice. Neuroscience 69:1075-1086.

Brennan PA, Schellinck HM, de la Riva C, Kendrick KM, Keverne EB (1998) Changes in neurotransmitter release in the main olfactory bulb following an olfactory conditioning procedure in mice. Neuroscience 87:583-590.

Breton-Provencher V, Lemasson M, Peralta MR 3rd, Saghatelyan A (2009) Interneurons produced in adulthood are required for the normal functioning of the olfactory bulb network and for the execution of selected olfactory behaviors. J Neurosci 29:15245-15257.

Brock O, Keller M, Veyrac A, Douhard Q, Bakker J (2010) Short term treatment with estradiol decreases the rate of newly generated cells in the subventricular zone and main olfactory bulb of adult female mice. Neuroscience 166:368-376.

Brunton PJ, Russell JA (2008) The expectant brain: adapting for motherhood. Nat Rev Neurosci 9:11-25.

Carleton A, Petreanu LT, Lansford R, Alvarez-Buylla A, Lledo PM (2003) Becoming a new neuron in the adult olfactory bulb. Nat Neurosci 6:507-518.

Carter CS, Altemus M, Chrousos GP (2001) Neuroendocrine and emotional changes in the post-partum period. Prog Brain Res 133:241-249.

Cohen L, Rothschild G, Mizrahi A (2011) Multisensory integration of natural odors and sounds in the auditory cortex. Neuron 72:357-369.

Dahlen JE, Jimenez DA, Gerkin RC, Urban NN (2011) Morphological analysis of activity-reduced adult-born neurons in the mouse olfactory bulb. Front Neurosci 5:66.

Dickinson C, Keverne EB (1988) Importance of noradrenergic mechanisms in the olfactory bulbs for the maternal behaviour of mice. Physiol Behav 43:313-316.

Feierstein CE, Lazarini F, Wagner S, Gabellec MM, de Chaumont F, OlivoMarin JC, Boussin FD, Lledo PM, Gheusi G (2010) Disruption of adult neurogenesis in the olfactory bulb affects social interaction but not maternal behavior. Front Behav Neurosci 4:176.

Furuta M, Bridges RS (2005) Gestation-induced cell proliferation in the rat brain. Brain Res Dev Brain Res 156:61-66.

Galindo-Leon EE, Lin FG, Liu RC (2009) Inhibitory plasticity in a lateral band improves cortical detection of natural vocalizations. Neuron 62:705-716.

Gandelman R, Zarrow MX, Denenberg VH, Myers M (1971) Olfactory bulb removal eliminates maternal behavior in the mouse. Science 171:210-211.

Gao Y, Strowbridge BW (2009) Long-term plasticity of excitatory inputs to granule cells in the rat olfactory bulb. Nat Neurosci 12:731-733.
Gross CG (2000) Neurogenesis in the adult brain: death of a dogma. Nat Rev Neurosci 1:67-73.

Grota LJ, Ader R (1969) Continuous recording of maternal behaviour in Rattus norvegicus. Animal Behav 17:722-729.

Grutzendler J, Kasthuri N, Gan WB (2002) Long-term dendritic spine stability in the adult cortex. Nature 420:812-816.

Guéneau G, Privat A, Drouet J, Court L (1982) Subgranular zone of the dentate gyrus of young rabbits as a secondary matrix. A high-resolution autoradiographic study. Dev Neurosci 5:345-358.

Hofer SB, Mrsic-Flogel TD, Bonhoeffer T, Hübener M (2009) Experience leaves a lasting structural trace in cortical circuits. Nature 457:313-317.

Holtmaat A, Svoboda K (2009) Experience-dependent structural synaptic plasticity in the mammalian brain. Nat Rev Neurosci 10:647-658.

Holtmaat AJ, Trachtenberg JT, Wilbrecht L, Shepherd GM, Zhang X, Knott GW, Svoboda K (2005) Transient and persistent dendritic spines in the neocortex in vivo. Neuron 45:279-291.

Holtmaat A, Bonhoeffer T, Chow DK, Chuckowree J, De Paola V, Hofer SB, Hübener M, Keck T, Knott G, Lee WC, Mostany R, Mrsic-Flogel TD, Nedivi E, Portera-Cailliau C, Svoboda K, Trachtenberg JT, Wilbrecht L (2009) Long-term, high-resolution imaging in the mouse neocortex through a chronic cranial window. Nat Protoc 4:1128-1144.

Hübener M, Bonhoeffer T (2010) Searching for engrams. Neuron 67: 363-371.

Isaacson JS, Strowbridge BW (1998) Olfactory reciprocal synapses: dendritic signaling in the CNS. Neuron 20:749-761.

Jahr CE, Nicoll RA (1980) Dendrodendritic inhibition: demonstration with intracellular recording. Science 207:1473-1475.

Keller M, Baum MJ, Brock O, Brennan PA, Bakker J (2009) The main and the accessory olfactory systems interact in the control of mate recognition and sexual behavior. Behav Brain Res 200:268-276.

Kelsch W, Lin CW, Lois C (2008) Sequential development of synapses in dendritic domains during adult neurogenesis. Proc Natl Acad Sci U S A 105:16803-16808.

Kelsch W, Lin CW, Mosley CP, Lois C (2009) A critical period for activitydependent synaptic development during olfactory bulb adult neurogenesis. J Neurosci 29:11852-11858.

Kendrick KM, Lévy F, Keverne EB (1992) Changes in the sensory processing of olfactory signals induced by birth in sleep. Science 256:833-836.

Kendrick KM, Guevara-Guzman R, Zorrilla J, Hinton MR, Broad KD, Mimmack M, Ohkura S (1997) Formation of olfactory memories mediated by nitric oxide. Nature 388:670-674.

Kinsley CH, Trainer R, Stafisso-Sandoz G, Quadros P, Marcus LK, Hearon C, Meyer EA, Hester N, Morgan M, Kozub FJ, Lambert KG (2006) Motherhood and the hormones of pregnancy modify concentrations of hippocampal neuronal dendritic spines. Horm Behav 49:131-142.

Kopec CD, Li B, Wei W, Boehm J, Malinow R (2006) Glutamate receptor exocytosis and spine enlargement during chemically induced long-term potentiation. J Neurosci 26:2000-2009.

Larsen CM, Grattan DR (2010) Prolactin-induced mitogenesis in the subventricular zone of the maternal brain during early pregnancy is essential for normal postpartum behavioral responses in the mother. Endocrinology 151:3805-3814.

Laurent G, Stopfer M, Friedrich RW, Rabinovich MI, Volkovskii A, Abarbanel HD (2001) Odor encoding as an active, dynamical process: experiments, computation, and theory. Annu Rev Neurosci 24:263-297.

Lazarini F, Lledo PM (2011) Is adult neurogenesis essential for olfaction? Trends Neurosci 34:20-30.

Leuner B, Gould E (2010) Dendritic growth in medial prefrontal cortex and cognitive flexibility are enhanced during the postpartum period. J Neurosci 30:13499-13503.

Leuner B, Glasper ER, Gould E (2010) Parenting and plasticity. Trends Neurosci 33:465-473.

Lévy F, Gervais R, Kindermann U, Orgeur P, Piketty V (1990) Importance of beta-noradrenergic receptors in the olfactory bulb of sheep for recognition of lambs. Behav Neurosci 104:464-469.

Lévy F, Gheusi G, Keller M (2011) Plasticity of the parental brain: a case for neurogenesis. J Neuroendocrinol 23:984-993.

Lin CW, Sim S, Ainsworth A, Okada M, Kelsch W, Lois C (2010) Genetically increased cell-intrinsic excitability enhances neuronal integration into adult brain circuits. Neuron 65:32-39.

Liu RC, Linden JF, Schreiner CE (2006) Improved cortical entrainment to 
infant communication calls in mothers compared with virgin mice. Eur J Neurosci 23:3087-3097.

Livneh Y, Mizrahi A (2011) Long-term changes in the morphology and synaptic distributions of adult-born neurons. J Comp Neurol 519:2212-2224.

Livneh Y, Mizrahi A (2012) Experience-dependent plasticity of mature adult-born neurons. Nat Neurosci 15:26-28.

Livneh Y, Feinstein N, Klein M, Mizrahi A (2009) Sensory input enhances synaptogenesis of adult-born neurons. J Neurosci 29:86-97.

Lledo PM, Alonso M, Grubb MS (2006) Adult neurogenesis and functional plasticity in neuronal circuits. Nat Rev Neurosci 7:179-193.

Lois C, Alvarez-Buylla A (1994) Long-distance neuronal migration in the adult mammalian brain. Science 264:1145-1148.

Mann PE, Bridges RS (2001) Lactogenic hormone regulation of maternal behavior. Prog Brain Res 133:251-262.

Margrie TW, Sakmann B, Urban NN (2001) Action potential propagation in mitral cell lateral dendrites is decremental and controls recurrent and lateral inhibition in the mammalian olfactory bulb. Proc Natl Acad Sci U S A 98:319-324.

Matsuzaki M, Honkura N, Ellis-Davies GC, Kasai H (2004) Structural basis of long-term potentiation in single dendritic spines. Nature 429:761-766.

Ming GL, Song H (2005) Adult neurogenesis in the mammalian central nervous system. Annu Rev Neurosci 28:223-250.

Mizrahi A (2007) Dendritic development and plasticity of adult-born neurons in the mouse olfactory bulb. Nat Neurosci 10:444-452.

Mizrahi A, Katz LC (2003) Dendritic stability in the adult olfactory bulb. Nat Neurosci 6:1201-1207.

Mori K, Takagi SF (1978) An intracellular study of dendrodendritic inhibitory synapses on mitral cells in the rabbit olfactory bulb. J Physiol 279:569-588.

Mori K, Nagao H, Yoshihara Y (1999) The olfactory bulb: coding and processing of odor molecule information. Science 286:711-715.

Noirot E (1969) Serial order of maternal responses in mice. Anim Behav 17:547-550.

Noirot E (1972) Ultrasounds and maternal behavior in small rodents. Dev Psychobiol 5:371-387.

Nusser Z, Kay LM, Laurent G, Homanics GE, Mody I (2001) Disruption of GABA(A) receptors on GABAergic interneurons leads to increased oscillatory power in the olfactory bulb network. J Neurophysiol 86:2823-2833.

Pawluski JL, Galea LA (2006) Hippocampal morphology is differentially affected by reproductive experience in the mother. J Neurobiol 66:71-81.

Pawluski JL, Barakauskas VE, Galea LA (2010) Pregnancy decreases oestrogen receptor alpha expression and pyknosis, but not cell proliferation or survival, in the hippocampus. J Neuroendocrinol 22:248-257.

Petreanu L, Alvarez-Buylla A (2002) Maturation and death of adult-born olfactory bulb granule neurons: role of olfaction. J Neurosci 22:61066113.
Rakic P, Bourgeois JP, Eckenhoff MF, Zecevic N, Goldman-Rakic PS (1986) Concurrent overproduction of synapses in diverse regions of the primate cerebral cortex. Science 232:232-235.

Roberts TF, Tschida KA, Klein ME, Mooney R (2010) Rapid spine stabilization and synaptic enhancement at the onset of behavioural learning. Nature 463:948-952.

Rochefort C, Gheusi G, Vincent JD, Lledo PM (2002) Enriched odor exposure increases the number of newborn neurons in the adult olfactory bulb and improves odor memory. J Neurosci 22:2679-2689.

Saghatelyan A, Roux P, Migliore M, Rochefort C, Desmaisons D, Charneau P, Shepherd GM, Lledo PM (2005) Activity-dependent adjustments of the inhibitory network in the olfactory bulb following early postnatal deprivation. Neuron 46:103-116.

Sakamoto M, Imayoshi I, Ohtsuka T, Yamaguchi M, Mori K, Kageyama R (2011) Continuous neurogenesis in the adult forebrain is required for innate olfactory responses. Proc Natl Acad Sci U S A 108:8479-8484.

Schaefer ML, Yamazaki K, Osada K, Restrepo D, Beauchamp GK (2002) Olfactory fingerprints for major histocompatibility complex-determined body odors II: relationship among odor maps, genetics, odor composition, and behavior. J Neurosci 22:9513-9521.

Seitz PF (1958) The maternal instinct in animal subjects. I. Psychosom Med 20:215-226.

Shepherd G, Chen WI, Greer CA (2004) Olfactory bulb. In: The synaptic organization of the brain (Shepherd G, ed), pp 165-216. Oxford, UK: Oxford UP.

Shingo T, Gregg C, Enwere E, Fujikawa H, Hassam R, Geary C, Cross JC, Weiss S (2003) Pregnancy-stimulated neurogenesis in the adult female forebrain mediated by prolactin. Science 299:117-120.

Urban NN (2002) Lateral inhibition in the olfactory bulb and in olfaction. Physiol Behav 77:607-612.

Urban NN, Arevian AC (2009) Computing with dendrodendritic synapses in the olfactory bulb. Ann N Y Acad Sci 1170:264-269.

Vandenbergh JG (1973) Effects of central and peripheral anosmia on reproduction of female mice. Physiol Behav 10:257-261.

Whitman MC, Greer CA (2007) Synaptic integration of adult-generated olfactory bulb granule cells: basal axodendritic centrifugal input precedes apical dendrodendritic local circuits. J Neurosci 27:9951-9961.

Yamaguchi M, Mori K (2005) Critical period for sensory experiencedependent survival of newly generated granule cells in the adult mouse olfactory bulb. Proc Natl Acad Sci U S A 102:9697-9702.

Yokoi M, Mori K, Nakanishi S (1995) Refinement of odor molecule tuning by dendrodendritic synaptic inhibition in the olfactory bulb. Proc Natl Acad Sci U S A 92:3371-3375.

Zuo Y, Lin A, Chang P, Gan WB (2005) Development of long-term dendritic spine stability in diverse regions of cerebral cortex. Neuron 46:181189. 